\title{
Goodness-of-fit Measures Based on the Mellin Transform for Beta Generalized Lifetime Data
}

\author{
Josimar Vasconcelos ${ }^{1}$, Renato Cintra ${ }^{2}$, and Abraão Nascimento ${ }^{2}$ \\ ${ }^{1}$ Federal Rural University of Pernambuco \\ ${ }^{2}$ Federal University of Pernambuco
}

April 8, 2021

\begin{abstract}
In recent years various probability models have been proposed for describing lifetime data. Increasing model flexibility is often sought as a means to better describe asymmetric and heavy tail distributions. Such extensions were pioneered by the beta-G family. However, efficient goodness-of-fit ( $\mathrm{GoF})$ measures for the beta-G distributions are sought. In this paper, we combine probability weighted moments (PWMs) and the Mellin transform (MT) in order to furnish new qualitative and quantitative GoF tools for model selection within the beta-G class. We derive PWMs for the Fr $\backslash$ ' $\{$ e $\}$ chet and Kumaraswamy distributions; and we provide expressions for the MT, and for the log-cumulants (LC) of the beta-Weibull, beta-Fr $\backslash$ ' $\{$ e $\}$ chet, beta-Kumaraswamy, and beta-log-logistic distributions. Subsequently, we construct LC diagrams and, based on the Hotelling's $\$ \mathrm{~T}^{\wedge} 2 \$$ statistic, we derive confidence ellipses for the LCs. Finally, the proposed GoF measures are applied on five real data sets in order to demonstrate their applicability.
\end{abstract}

\section{Hosted file}

GoF_MTA.pdf available at https://authorea.com/users/406625/articles/517207-goodness-of-fitmeasures-based-on-the-mellin-transform-for-beta-generalized-lifetime-data 
figures/GoFBF/GoFBF-eps-converted-to.pdf 
figures/GoFBLL/GoFBLL-eps-converted-to.pdf 
figures/GoFBKW/GoFBKW-eps-converted-to.pdf 
figures/GoFBW/GoFBW-eps-converted-to.pdf 
figures/betaF/betaF-eps-converted-to.pdf 
figures/betaW/betaW-eps-converted-to.pdf 
figures/betaKW/betaKW-eps-converted-to.pdf 
figures/betaLL/betaLL-eps-converted-to.pdf 
figures/ellipsesBR/ellipsesBR-eps-converted-to.pdf 
figures/ellipsesGU/ellipsesGU-eps-converted-to.pdf 
figures/ellipsesST/ellipsesST-eps-converted-to.pdf 
figures/ellipsesAI/ellipsesAI-eps-converted-to.pdf 
figures/ellipsesFL/ellipsesFL-eps-converted-to.pdf 
figures/betaG-diagram/betaG-diagram-eps-converted-to.pdf 
figures/betaG-app1/betaG-app1-eps-converted-to.pdf 